\title{
Development of microtubule capping structures in ciliated epithelial
}

\section{cells}

\author{
R. W. PORTMAN, E. L. LeCLUYSE and W. L. DENTLER \\ Department of Physiology and Cell Biologv, Universtty of Kansas, Laturence, KS 66045, USA
}

\section{Summary}

Although capping structures are present at the tips of microtubules in both growing cilia and mature cilia, previous work has not determined the time of cap formation. The results reported here reveal that the large caps of mature palate cilia appear in cilia with lengths as short as $1.75 \mu \mathrm{m}$. In the growing palate cilium, a diskshaped plate is formed at the tip during the first $\mu \mathrm{m}$ of growth. As the cilium elongates to $1.5-2.0 \mu \mathrm{m}$, a small plate forms underneath the disk-shaped plate that gives an asymmetrical appearance to the whole cap structure. The structure of the cap is complete in cilia longer than $2.0 \mu \mathrm{m}$. The hair-like structures that form the extraciliary crown appear on the membrane at the ciliary tip at the same time as the mature cap is forming. The formation of a cap structure is discussed in relation to microtubule assembly during ciliogenesis.

Key words: cilia, flagella, microtubules, basal bodies, ciliogenesis.

\section{Introduction}

Capping structures are attached to the distal tips of central and outer doublet A-tubules in most, if not all, cilia and flagella (Dentler, 1980, 1986; Dentler \& Rosenbaum, 1977). Studies of growing cilia and flagella in the protozoans Tetrahymena and Chlamydomonas revealed that the capping structures are fully formed and associated with axonemes when cilia are as short as $4 \mu \mathrm{m}$ (Dentler, 1980). Both the distal filaments, attached to each A-tubule, and the central microtubule cap, attached to both of the central tubules, remain attached to the microtubules as the cilia grow (Dentler, 1980). Since these structures are at the sites at which tubulin is added to the growing microtubules (Rosenbaum et al. 1969; Witman, 1975), and since the capping structures are tightly bound to the ends of the microtubules (Dentler \& LeCluyse, $1982 a, b)$, we suggested that these structures are involved in the assembly of ciliary microtubules (Dentler, 1980).

Since the capping structures appear in cilia as short as $4 \mu \mathrm{m}$, they must be one of the first structures to be formed after the basal body docks with the plasma membrane and microtubule growth is initiated (Dentler, 1980). Proof of this proposal is difficult. The capping structures in protozoan cilia are very Journal of Cell Science 87, 85-94 (1987) Printed in Great Britam (C) The Company of Biologists Limited 1987 small and are best visualized in negatively stained axonemes. However, it is not possible to obtain good negatively stained axonemes within $2-3 \mu \mathrm{m}$ of the basal body owing to the size of the cell body. Studies of thin-sectioned Tetrahymena cells with regenerating cilia were carried out, but it was difficult to determine precisely when the capping structures first appeared in the growing cilia (Dentler, 1980).

In comparison with protozoans, the capping structures in vertebrate epithelial cilia are quite prominent and are easily resolved in thin sections (Dentler \& LeCluyse, 1982a; LeCluyse \& Dentler, 1984). In these cilia, a large cap is attached to the central tubules and to each of the A-tubules by plug structures inserted into the lumen of each microtubule in a manner similar in appearance to the attachment of capping structures to protozoan microtubules (Dentler \& LeCluyse, 1982a; LeCluyse \& Dentler, 1984). Since the large cap is easily identified in thin sections and since the density of cilia along ciliated epithelial tissue permits a large number of cilia to be examined in a reasonable number of thin sections, this tissue is ideal for a study of the time and position of cap formation.

The palate of the frog Bombina orientalis is chosen for several reasons. (1) The ciliary caps are not only prominent but are composed of two plates of unequal 
size positioned over specific doublet microtubules (LeCluyse \& Dentler, 1984); this asymmetrical cap permits us to identify the time at which the cap appears during ciliogenesis as well as revealing the time at which the cap becomes positioned on specific microtubules. (2) Most of the initiation and growth of cilia in the Bombina palate occurs within a short period of time during metamorphosis (LeCluyse et al. 1985);
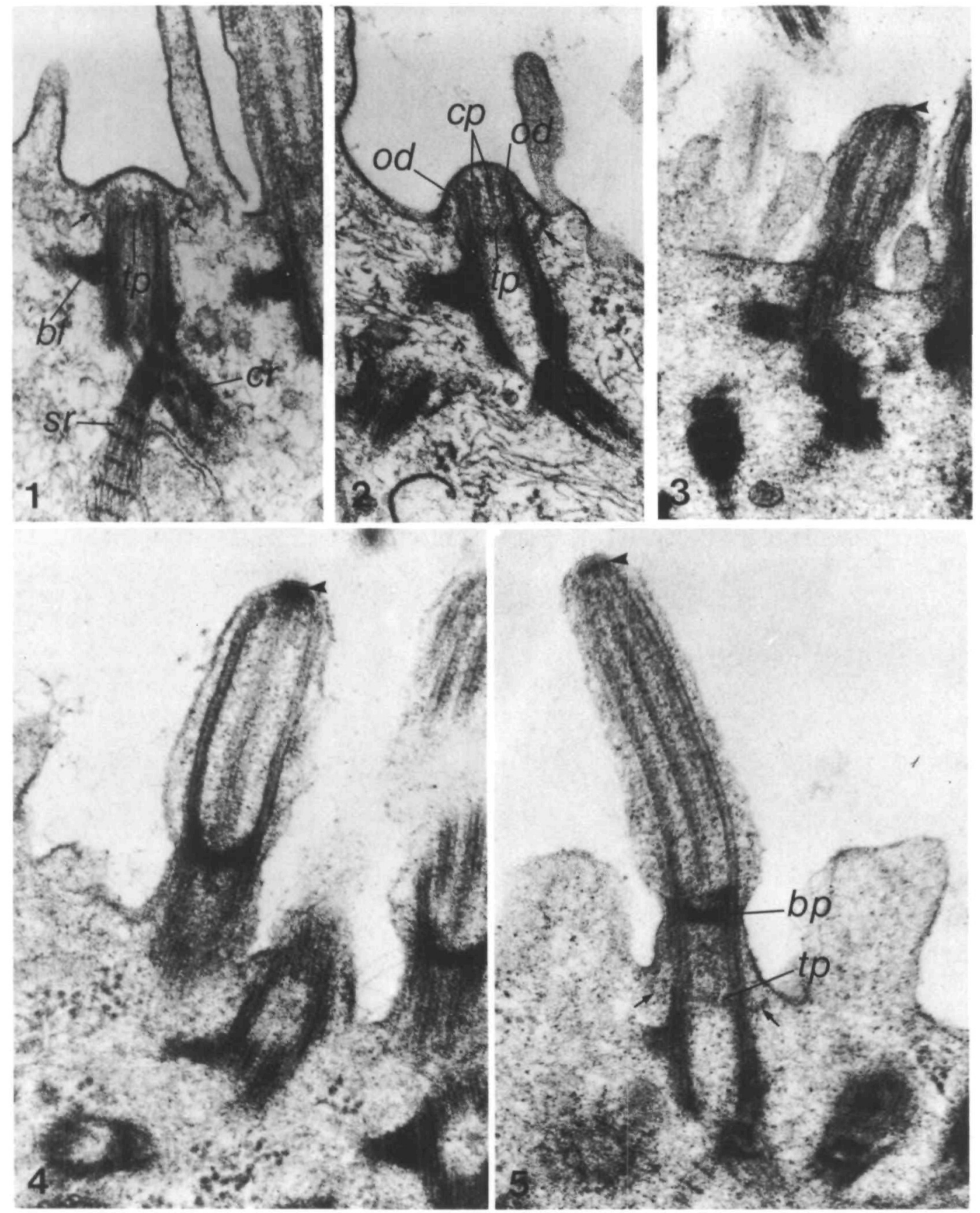

Figs 1-5. Cilia with lengths up to $1 \mu \mathrm{m}$ reveal the development of a cap structure. All figures, magnification: $\times 52500$.

Fig. 1. A basal body has a well-defined striated rootlet $(s r)$, ciliary root $(c r)$, basal foot $(b f)$, transitional fibrils (small arrows), and a transverse plate $(t p)$, before formation of a cilium.

Fig. 2. A short cilium, $0 \cdot 17 \mu \mathrm{m}$ length, has outer doublet $(o d)$ and central-pair $(c p)$ microtubules, which extend from the basal body to the ciliary tip. The transverse plate is identified by tp. Small arrows point out transitional fibrils.

Fig. 3. A cilium, $0.5 \mu \mathrm{m}$ length, shows amorphous electron-dense material (arrowhead) forming at the tip.

Fig. 4. A cilium, $0.88 \mu \mathrm{m}$ in length, shows a disk-shaped cap structure at its tip (arrowhead).

Fig. 5. A cilium, $1 \cdot 0 \mu \mathrm{m}$ long, shows a disk-shaped cap structure at its tip (arrowhead). Arrows show position of transitional fibrils between basal body and plasma membrane. The positions of the basal plate and the transverse plate are noted by $b p$ and $t p$, respectively. 
this increases the number of growing cilia that can be observed in a reasonable number of thin sections. (3) Cilia grow asynchronously over the surface of the frog palate so that any individual palate cell can contain some fully grown as well as growing cilia; this ensures that the capping structures are preserved in each preparation fixed for electron microscopy. (4) Bombina tadpoles are easily obtained and raised in the laboratory.

The results reported here show that the capping structures in palate cilia are fully formed by the time each cilium has grown $1 \cdot 75 \mu \mathrm{m}$. Although amorphous material is seen between the distal tips of microtubules and the ciliary membrane in cilia shorter than $0.75 \mu \mathrm{m}$, the cap is poorly defined and positive identification of the capping structures cannot be made. By the time the cilium has grown $1.75-2.0 \mu \mathrm{m}$, the cap and asymmetrical arrangement of plates are completely formed, and the cap is attached to the ciliary crown hairs that extend from the external face of the ciliary membrane. By these structural criteria, we conclude that the capping structures in epithelial cilia are completely formed during the first $2 \mu \mathrm{m}$ of ciliary growth and remain attached to the distal tips of the assembling microtubules throughout ciliary assembly. A preliminary report of these data has been published in abstract form (Portman \& Dentler, 1986).

\section{Materlals and methods}

Bombina orientalis tadpoles were raised in the laboratory and were killed during metamorphosis as the tails were regressing. The developmental stage of each tadpole was determined using the classification scheme of Taylor \& Kollros (1946). To obtain palates, the metamorphosing frogs were decapitated and the lower jaw was separated from the upper jaw. The upper jaw was washed in Holtfreter's solution $\left(60.14 \mathrm{~mm}-\mathrm{NaCl}, \quad 0.24 \mathrm{~mm}-\mathrm{NaHCO}_{3}, \quad 0.90 \mathrm{~mm}\right.$ $\mathrm{CaCl}_{2}, 0.67 \mathrm{~mm}-\mathrm{KCl}$ ) for $10-15 \mathrm{~min}$ prior to fixation to remove palate mucus.

The palates were fixed by immersion in $1 \% \mathrm{OsO}_{4}, 2 \%$ glutaraldehyde, $150 \mathrm{~mm}$-sodium phosphate, $\mathrm{pH} 7 \cdot 4$, for $20-30 \mathrm{~min}$ at $4^{\circ} \mathrm{C}$ (Omoto \& Kung, 1980). The fixative was removed, and the upper jaw rinsed in distilled water for $5 \mathrm{~min}$ to remove phosphate buffer prior to overnight immersion in $1 \%$ uranyl acetate. The palate epithelium was then dissected away from the skull and soft brain tissue, and the palate was cut into quarters. The pieces of palate epithelium were dehydrated in an ascending acetone series and embedded in Spurr resin (Spurr, 1969). Silver sections were obtained with a diamond knife, picked up on 300 mesh grids, and stained with aqueous $2 \%$ uranyl acetate and lead citrate (Venable \& Coggeshall, 1965) for $10 \mathrm{~min}$ and $2 \mathrm{~min}$, respectively. Sections were examined and photographed using a Philips 300 electron microscope.

\section{Results}

Thin sections of developing palate cilia reveal all stages in ciliogenesis from the time of basal body attachment to the plasma membrane to the growth of the ciliary microtubules. Figs 1-5 show the development of palate cilia up to $1.0 \mu \mathrm{m}$ in length. Very short cilia are connected to basal bodies, which show a well-defined ciliary root, striated rootlet and basal foot (Figs 1,2). In addition, transitional fibrils extend outward from the upper third of the basal body to the plasma

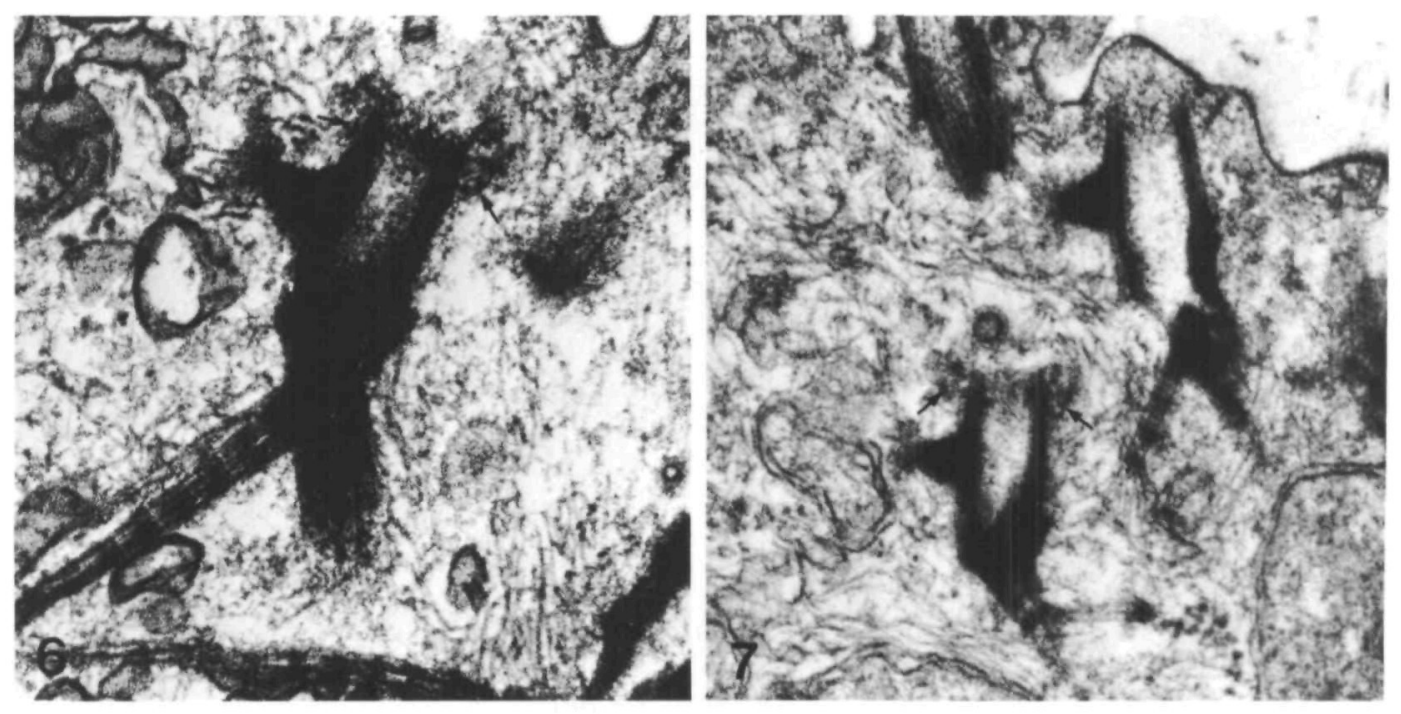

Figs 6-7. Basal bodies before docking with membrane. Both figures, $\times 52500$.

Fig. 6. The basal body has all its associated structures (see Fig. 1) attached before it has docked with membrane. Transitional fibrils (small arrows) seen at the distal end of the basal body.

Fig. 7. Transitional fibrils (small arrows) are shown on the basal body before docking. A basal body that has recently docked at the plasma membrane (upper right) shows all associated structures plus transitional fibrils. 
membrane on both sides of the basal body (small arrows, Figs 1, 2, 5; Pitelka, 1974). A plate of electron-dense material, called the terminal plate (Pitelka, 1974), extends across the lumen of the distal end of the basal body (Figs 1, 2, 5). The basal bodyassociated structures (rootlets, basal foot, transitional fibrils and terminal plate) are present prior to the time that the basal body attaches to the plasma membrane (see Figs 6,7). As the ciliary microtubules increase in length, a basal plate forms at the proximal ends of the central-pair microtubules (Fig. 5). The basal plate is more electron-dense than the terminal plate and is approximately $0.25 \mu \mathrm{m}$ distal to the terminal plate (see the structures labelled $t p$ and $b p$, Fig. 5). The

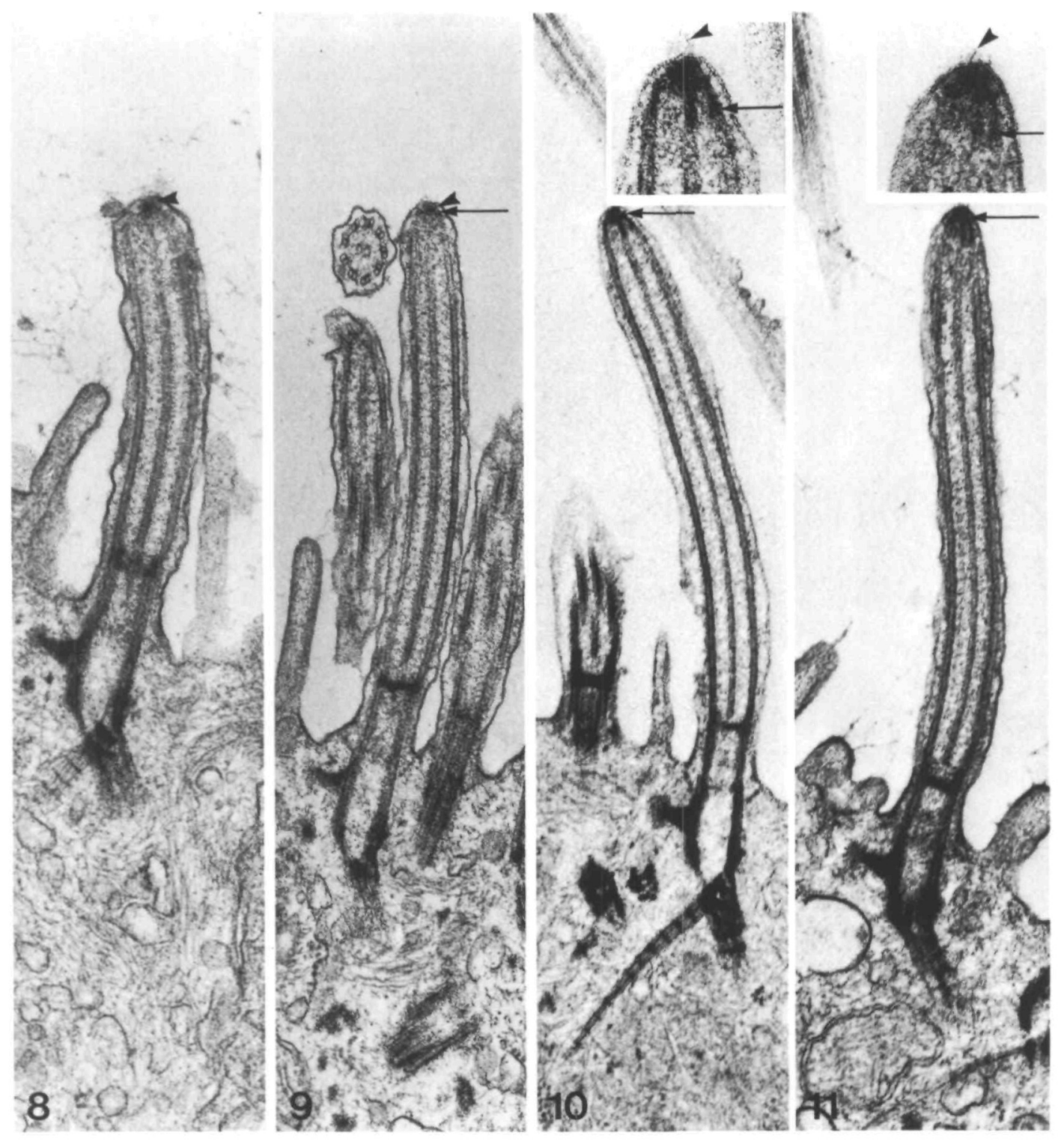

Figs 8-11. The further development of cap structures occurs in cilia with lengths between 1.5 and $2 \cdot 0 \mu \mathrm{m}$. All figures, $\times 40000$; insets, $\times 75600$.

Fig. 8. The cap structure (arrowhead) in a cilium of $1.45 \mu \mathrm{m}$ is similar to structures at the tips of shorter cilia (see Figs 4-5).

Fig. 9. A cilium with a length of $\approx 1 \cdot 75 \mu \mathrm{m}$ shows the early development of a lower plate (small arrow) on the right side of the cap structure (arrowhead).

Fig. 10. A cilium with a length of $1.90 \mu \mathrm{m}$ shows further development of the lower plate and plug extensions into microtubules beneath both plates. The lower plate (small arrow) is tilted downwards relative to the upper shelf. Inset shows the ciliary crown (arrowhead), plug extensions (arrow) as well as the position of the lower plate. Plug extensions have lengths of up to $\approx 50 \mathrm{~nm}$.

Fig. 11. A $2 \cdot 0 \mu \mathrm{m}$ long cilium shows the development of the lower plate (small arrow) and plug extensions (inset, arrow). A ciliary crown (arrowhead) is shown in the inset. 
Table 1. Presence of asymmetrical capping structures in cilia of different lengths

\begin{tabular}{ccc}
\hline $\begin{array}{c}\text { Ciliary } \\
\text { length }(\mu \mathrm{m})\end{array}$ & $\begin{array}{c}\text { Percentage of } \\
\text { cilia with caps }\end{array}$ & $\begin{array}{c}\text { Percentage of } \\
\text { capped cilia with } \\
\text { asymmetrical caps }\end{array}$ \\
\hline $0 \cdot 00-0 \cdot 25$ & $0(12)$ & 0 \\
$0 \cdot 25-0 \cdot 50$ & $0(8)$ & 0 \\
$0 \cdot 50-0 \cdot 75$ & $41(17)$ & 0 \\
$0 \cdot 75-1 \cdot 00$ & $56(16)$ & 0 \\
$1 \cdot 00-1 \cdot 25$ & $50(8)$ & 0 \\
$1 \cdot 25-1 \cdot 50$ & $82(11)$ & 0 \\
$1 \cdot 50-1 \cdot 75$ & $85(13)$ & 18 \\
$1 \cdot 75-2 \cdot 00$ & $100(8)$ & 50 \\
$2 \cdot 00-2 \cdot 50$ & $100(12)$ & 83 \\
$2 \cdot 50-3 \cdot 50$ & $100(13)$ & 69 \\
$3 \cdot 50-6 \cdot 00$ & $100(19)$ & 100
\end{tabular}

The centre column shows the percentage of cilia with caps at each ciliary length, and the right-hand column shows the percentage of capped cilia that contain clear views of the asymmetrical arrangement of plates typical of fully grown Bombina cilia. The number of cilia scored for each measured length is given in parenthesis. Since the plates lie in the same plane as the basal feet attached to each basal body, the capping structures are only counted from thin sections of cilia in which the basal fect are visiblc. Capping structures are seen with increasing frequency in culia greater than $0.5 \mu \mathrm{m}$ in length. The asymmetrically positioned plates in the caps, indicative of 'mature' caps are observed with increasing frequency in cilia greater than $1.75 \mu \mathrm{m}$.

proximal ends of central-pair microtubules appear to end at the terminal plate in cilia equal to or less than $0.5 \mu \mathrm{m}$ in length (Fig. 2).

Electron-dense material similar in appearance to the capping structures can be seen between the microtubule tips and the ciliary membrane in cilia as short as $0.5 \mu \mathrm{m}$ (arrowhead in Fig. 3). These structures are seen with increasing frequency in cilia between $0.5 \mu \mathrm{m}$ and $1.75 \mu \mathrm{m}$ long (arrowheads, Figs 3, 4, 5, 8, 9). Table 1 summarizes these data obtained from many thin sections.

The cap structure undergoes further changes in its form as the cilium grows from $1.5 \mu \mathrm{m}$ to $6 \mu \mathrm{m}$. Our previous studies showed that the capping structures in fully grown palate cilia are composed of two different size plates. The large plate is attached to doublet microtubules nos 4-7 while the smaller plate is attached to doublet nos $1,2,3,8,9$, as well as to the central microtubules (LeCluyse \& Dentler, 1984). Since the plates are asymmetrically positioned in the cap, they can be seen only in those cilia sectioned in a plane that also contains the basal feet (Figs 10-16; LeCluyse \& Dentler, 1984).

The asymmetrical cap structures are visible in cilia with lengths between $1 \cdot 75$ and $2 \cdot 0 \mu \mathrm{m}$ (Figs 9, 10, 11). The frequency of cap asymmetry increases in cilia between 1.75 and $2.5 \mu \mathrm{m}$ long so that virtually all cilia greater than $2 \cdot 5 \mu \mathrm{m}$ contain an asymmetrical cap (Figs
12-16; Table 1). Since the asymmetrical structure of the cap is only visible in certain orientations of the cilia, it is likely that all cilia longer than $2-2.5 \mu \mathrm{m}$ long have fully formed capping structures identical to those in fully grown cilia.

The first appearance of the two plates is defined by the presence of amorphous electron-dense material underneath the disk-shaped cap described in shorter cilia (arrows, Figs 9, 10). The downward tilt of the lower shelf relative to the upper shelf suggests that the lower shelf is not yet positioned within the whole cap structure (inset, Fig. 10). Plug structures, similar to those reported previously in mature palate cilia (LeCluyse \& Dentler, 1984) and protozoan cilia (Dentler, 1981), extend downwards from the developing cap and insert into the lumen of each A-tubule and each central-pair microtubule. The plug structures are as long as $50 \mathrm{~nm}$ (insets, Figs 10, 11).

Cilia longer than $3.5 \mu \mathrm{m}$ show bending patterns characteristic of mature cilia that are 'instantly fixed' (Omoto \& Kung, 1980) during their ciliary beat (Figs $14,15,16)$. Some cilia show tilting of the whole cap structure beneath the tip of the ciliary membrane (Fig. 15).

A ciliary crown, first described by Dirksen \& Satir (1972), consists of rows of fine bristles above the cap structure and ciliary membrane (see LeCluyse \& Dentler, 1984). The ciliary crown appears at the ciliary tip at the same time as the asymmetry is first apparent in the cap (small arrowheads, insets Figs 10, $11,14)$. The formation of the crown, therefore, occurs concomitant with the formation of a complete capping structure. Although the ciliary crown is reported at the tips of mature cilia from many different types of epithelia (see Dentler, 1981), this is the first demonstration that the crown hairs are present in growing cilia.

\section{Discussion}

Previous papers on studies of ciliogenesis in metazoans described centriole formation in differentiating epithelium from mouse oviduct (Dirksen, 1971), Rhesus monkey oviduct (Anderson \& Brenner, 1971), rat trachea (Dirksen \& Crocker, 1965), and chick trachea (Kalnins \& Porter, 1969). The formation of cilia in multiciliated epithelial cells has been described for only a few tissues, including tracheal epithelium from Xenopus embryos (Steinman, 1968), tracheal epithelium of the rat (Sorokin, 1968), and the tubular nephron of the lamprey (Youson, 1982). With the discovery of microtubule capping structures in metazoan as well as protozoan cilia (Dentler \& LeCluyse, 1982a; LeCluyse \& Dentler, 1984) and the observation that these structures remained bound to the distal ends of growing microtubules during most of 
ciliary growth (Dentler, 1980), it was important to reexamine the process of ciliogenesis to determine the site and timing of cap formation. Metazoan epithelial cilia are ideal for this study because their capping structures are prominent and well stained for examination in thin sections.

The results reported here show that the capping structures are neither associated with the basal body nor with the ciliary membrane during the initial stages of attachment between these organelles. Shortly after attachment of the basal body to the membrane, a mass of amorphous granular material gradually collects at the tips of the growing microtubules. When the ciliary microtubules are $0.75-1.5 \mu \mathrm{m}$ long (Table 1 ), however, a cap-like structure is seen within the amorphous material. Similar to earlier observations of Xenopus cilia by Steinman (1968), the dense material in Bombina palate cilia begins to differentiate into a multilayered cap. These layers eventually form the two plates of the asymmetrical cap previously shown
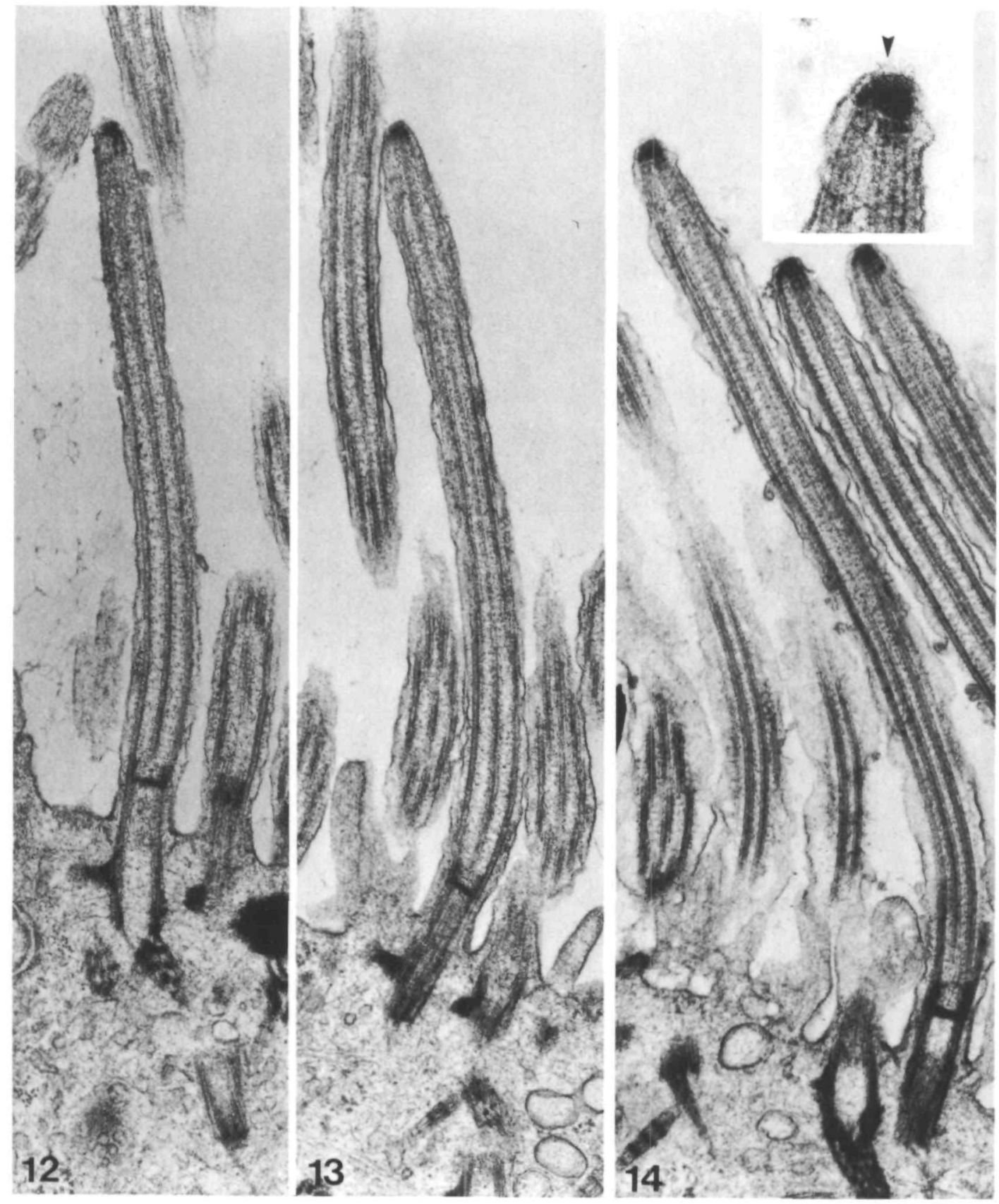

Figs 12-14. The tips of cilia show additional development of the cap. All Figs, $\times 40000 ;$ insets, $\times 75600$.

Figs 12, 13. These cilia with lengths of 2.5 and $2.95 \mu \mathrm{m}$, respectively, have a lower plate and plug extensions into central-pair and outer doublet microtubules.

Fig. 14. A cilium with a length of $3.47 \mu \mathrm{m}$ has both plates, and cap asymmetry is pointing to the right. Inset shows ciliary crown (arrowhead) as well as cap asymmetry. 
both in Bombina and in Xenopus cilia (LeCluyse \& Dentler, 1984; LeCluyse et al. 1985). The plates are linked to the microtubules by short plugs that insert into the lumen of each A-tubule and central microtubule. Together these structures form a capplug complex that binds to the microtubule tips

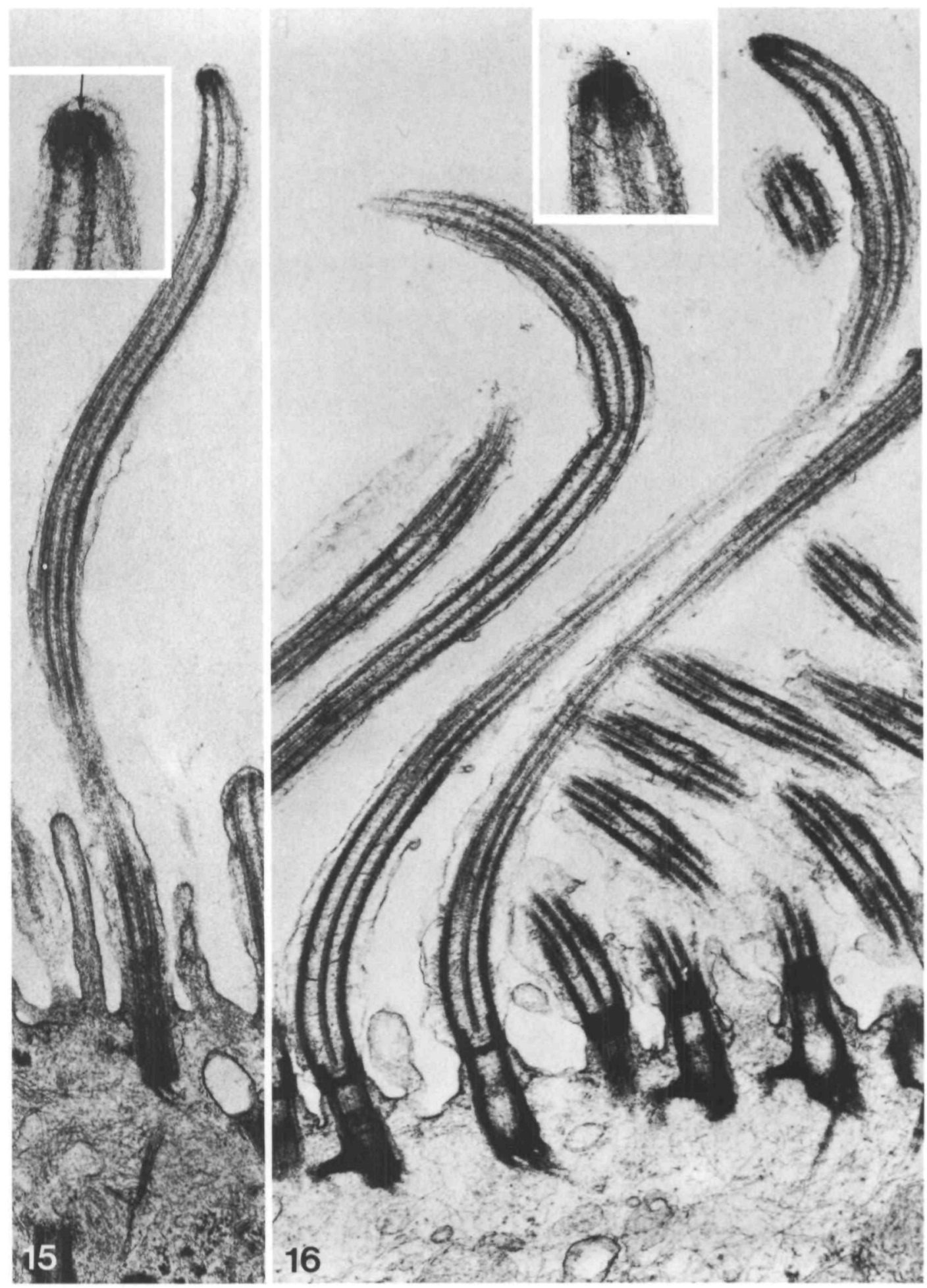

Figs 15-16. Cilia with lengths between 4.00 and 5.00 $\mu \mathrm{m}$. Both Figs, $\times 40000$; insets, $\times 81900$.

Fig. 15. A cilium of $3.9 \mu \mathrm{m}$ shows more bending than shorter cilia. Inset shows a cap tilted upwards under the ciliary membrane. Arrow points to lower plate.

Fig. 16. A cilium of $5 \cdot 1 \mu \mathrm{m}$ shows additional bending and a cap structure with the asymmetry pointing upwards. Inset shows lower plate pointing to the right. 
(Dentler, 1980; LeCluyse \& Dentler, 1984; Dentler \& LeCluyse, 1982a; Dentler, 1984).

The assembly of cap-plug complex and asymmetric placement of the plate structures characteristic of fully grown palate cilia occur early in development when the cilia reach $1 \cdot 75-2 \cdot 00 \mu \mathrm{m}$ (Table 1 ), although wellformed caps can be found in cilia as short as $0.75 \mu \mathrm{m}$. Once the cap is formed, it remains connected to the ends of microtubules throughout ciliary growth. These results correlate well with previous studies of capping structures in Chlamydomonas and Tetrahymena cilia, in which the capping structures are fully formed by the time the cilia are $3-4 \mu \mathrm{m}$ long and remain attached to the microtubules throughout ciliary growth (Dentler \& Rosenbaum, 1977; Dentler, 1980).

Although the results reported here reveal that the capping structures are assembled during the initial stages of ciliogenesis, it remains unclear precisely when the components of the caps are first assembled. We do know that a basal body will not initiate ciliary microtubule assembly until it has attached to the plasma membrane. Isolated basal bodies and centrioles can initiate the assembly of single cytoplasmic microtubules in vitro (Snell et al. 1974; Rosenbaum et al. 1975; Gould \& Borisy, 1977) and in vivo (Heideman et al. 1977), and single microtubules are occasionally observed to be attached to basal body-like centrioles in situ (Krishan \& Buck, 1965). However, the microtubules initiated by isolated basal bodies or centrioles were typical cytoplasmic microtubules and not the doublet microtubules found in cilia and eukaryotic flagella. The nucleated assembly of ciliary microtubules may, therefore, be regulated by some factor(s) associated with the docking of the basal body to the plasma membrane. The results of both in vivo and in vitro experiments suggest that initiation of microtubule assembly may require the release of some inhibitory factor from the ends of basal body microtubules before the attachment of microtubule capping structures. One function of the proteins or other factors associated with the capping structures may be to release the postulated inhibitor and initiate microtubule assembly. Another function may be to direct the assembly of ciliary microtubules, since only single (cytoplasmic) microtubules are assembled in vivo (Krishan \& Buck, 1965) or in vitro (Snell et al. 1974; Gould \& Borisy, 1977) onto the ends of basal bodies and centrioles that lack the capping structures. The results reported here show that the cap structures are formed within the first two micrometres of ciliary growth, but leave open the possibility that capping proteins may join with the basal bodies prior to the formation of the prominent caps seen in thin-sectioned or negatively stained cilia. Although it is unlikely that morphological studies alone will reveal the initial presence of capping structures or capping structure proteins, studies with antibodies directed against capping structures may be useful in future studies. Recent progress has been made in the isolation of capping structures (Suprenant \& Dentler, 1986) and, hopefully, antibodies against these structures will be obtained within the near future.

The axonemal microtubules of very short palate cilia appear more electron-opaque and less well arranged than do microtubules of fully grown cilia in Bombina. Some microtubules in the short cilia are difficult to identify (see Figs 1, 2). This problem has been discussed previously in studies of flagellar development in the amoeboflagellate Naegleria and ciliary development in neuroepithelial cells of chick embryos (Dingle \& Fulton, 1966; Sotelo \& Trujillo-Cenoz, 1958). The walls of axonemal microtubules become more electron-dense when the cilia reach a length at which the cap structure and a well-defined basal plate are formed. It may be that short microtubules are unstable but once the capping structures are assembled onto the ends of the microtubules, the tubulin within the microtubules may be modified to form a more stable polymer, perhaps, by acetylation as has been shown in Chlamydomonas (L'Hernault \& Rosenbaum, 1983; Piperno \& Fuller, 1985). Alternatively, the capping structures may facilitate the attachment of proteins (dynein, radial spoke proteins, etc.) to the ciliary microtubules that will increase microtubule stability.

In addition to their attachment to the ciliary microtubules, the capping structures in many but not all epithelial cilia (Dentler \& LeCluyse, 1982a) are linked, through the membrane, to a complex of extraciliary hairs, called the ciliary crown (Dirksen \& Satir, 1972; Kuhn \& Engleman, 1978; Dentler, 1981; LeCluyse \& Dentler, 1984). Although Dirksen \& Satir (1972) initially reported that the crown hairs appeared only on fully grown cilia, the results reported here showed that the crown was formed at the same time as the capping structure, since the crown and cap were found on virtually all cilia longer than $1-2 \mu \mathrm{m}$. The attachment of the cap to the membrane and to the crown may help stabilize or position the cap at the ciliary tip as the ciliary microtubules grow out to their final lengths.

We thank Drs Kathy Suprenant and Paul Burton for their helpful criticism on the manuscript. We also thank Scott Robinson and Dr Sally Frost for providing us with Bombina orientalis tadpoles and Ms Lorainne Hammer for technical assistance. This work was supported by grant from the National Institutes of Health (GM 32556). 


\section{References}

Anderson, R. G. W. \& Brenner, R. M. (1971). The formation of basal bodies (centrioles) in the rhesus monkey oviduct. F. Cell Biol. 50, 10-34.

DeNTLER, W. L. (1980). Structures linking the tips of ciliary and flagellar microtubules to the membrane. 7. Cell Sci. 42, 207-220.

DENTLER, W. L. (1981). Microtubule-membrane interactions in cilia and flagella. Int. Rev. Cytol. 72, $1-47$.

Dentler, W. L. (1984). Attachment of the cap to the central microtubules of Tetrahymena cilia. F. Cell Sci. 66, 167-173.

DENTLER, W. L. (1986). Isolation of capped cilia from bovine trachea and the effect of caps on microtubule assembly. I. Cell Biol. 103, 279a.

Dentler, W. L. \& LeCluyse, E. L. (1982a). Microtubule capping structures at the tips of tracheal c1lia: evidence for their firm attachment during ciliary bend formation and the restriction of microtubule sliding. Cell Motil. 2, 549-572.

Dentler, W. L. \& LeCluyse, E. L. (1982b). The effects of structures attached to the tips of tracheal ciliary microtubules on the nucleation of microtubule assembly in vitro. Cell Motil. (Suppl.) 1, 13-18.

Dentler, W. L. \& Rosenbaum, J. L. (1977). Flagellar elongation and shortening in Chlamydomonas. III. Structures attached to the tips of flagellar microtubules and their relationship to the directionality of fagellar microtubule assembly. F. Cell Biol. 74, 749-759.

Dingle, A. D. \& Fulton, C. (1966). Development of the flagellar apparatus of Naegleria. J. Cell Biol. 31, 43-54.

DiRKSEN, E. R. (1971). Centriole morphogenesis in developing ciliated epithelium of the mouse oviduct. 7. Cell Biol. 51, 286-302.

Dirksen, E. R. \& Crocker, T. T. (1965). Centriole replication in differentiating ciliated cells of mammalian respiratory epithelium. An electron microscope study. J. Microsc. 5, 629-644.

Dirksen, E. R. \& SATIR, P. (1972). Ciliary activity in the mouse oviduct as studied by transmission and scanning electron microscopy. Tissue $\mathbb{\sigma}^{\circ}$ Cell 4, 389-404.

Gould, R. R. \& BoRISY, G. G. (1977). The pericentriolar material in chinese hamster ovary cells nucleates microtubule formation. J. Cell Biol. 73, 601-615.

Heidemann, S. R., Sander, G. \& Kirschner, M. W. (1977). Evidence for a functional role of RNA in centrioles. Cell 10, 337-350.

Kalnins, V. I. \& Porter, K. R. (1969). Centriole replication during ciliogenesis in chick tracheal epithelium. Z. Zellforsch. mikrosk. Anat. 100, 1-30.

Krishan, A. \& Buck, R. C. (1965). Structure of the mitotic spindle in L-strain fibroblasts. J. Cell Biol. 24, 433-444.

Kunn, C. \& Engleman, W. (1978). The structure of the tips of mammalian respiratory cilia. Cell Tiss. Res. 186, 491-498.

LeCluyse, E. L. \& Dentler, W. L. (1984). Asymmetrical microtubule capping structures in frog palate cilia. 7 . Ultrasiruct. Res. 86, 75-85.
LeCluyse, E. L., Frost, S. K. \& Dentler, W. L. (1985). Development and ciliation of the palate in two frogs, Bombina and Xenopus; a comparative study. Tissue Eீ Cell 17, 853-864.

L'Hernault, S. W. \& Rosenbaum, J. L. (1983). Chlamydomonas $\alpha$-tubulin is posttranslationally modified in the flagella during flagella assembly. Y. Cell Biol. 97, 258-263.

Омото, C. K. \& KunG, C. (1980). Rotation and twist of the central pair microtubules in the cilia of Paramecium. 7. Cell Biol. 87, 33-46.

Piperno, G. \& Fuller, M. T. (1985). Monoclonal antibodies specific for an acetylated form of $\alpha$-tubulin recognize the antigen in cilia and flagella from a variety of organisms. Y. Cell Biol. 101, 2085-2094.

Pitelka, D. R. (1974). Basal bodies and root structures. In Cilia and Flagella (ed. M. A. Sleigh), pp. 437-469. New York, London: Academic Press.

Portman, R. W. \& Dentler, W. L. (1986). Development of asymmetrical capping structures and orientation of basal bodies during ciliogenesis on palate epithelium from Bombina orientalis. F. Cell Biol. 103, 279a

Rosenbaum, J. L., Binder, L. I., Granett, S., Dentler, W. L., Snell, W., Sloboda, R. \& Haimo, L. (1975). Directionality and rate of assembly of chick brain tubulin onto pieces of neurotubules, flagellar axonemes, and basal bodies. Ann. N.Y. Acad. Sci. 253, 147-177.

Rosenbaum, J. L., Moulder, J. E. \& Ringo, D. L. (1969). Flagellar elongation and shortening in Chlamydomonas. The use of cycloheximide and colchicine to study the synthesis and assembly of flagellar proteins. F. Cell Biol. 41, 600-619.

Snell, W. J., Dentler, W. L., Haimo, L., Binder, L. I. \& Rosenbaum, J. L. (1974). Assembly of chick brain tubulin onto isolated basal bodies of Chlamydomonas reinhardtii. Science 185, 357-360.

SOROKIN, S. (1968). Reconstruction of centriole formation and ciliogenesis in mammalian lungs. $\mathcal{F}$. Cell Sci. 3, 207-230.

Sotello, J. R. \& Trujillo-Cenoz, O. (1958). Electron microscope study on the epithelium of ciliary components of the neural epithelium of the chick embryo. Z. Zellforsch. mikrosk. Anat. 49, 1-12.

SpURR, A. R. (1969). A low viscosity epoxy resin embedding medium for electron microscopy. 7. Ultrastruct. Res. 26, 31-43.

StEInMan, R. M. (1968). An electron microscope study of ciliogenesis in developing epidermis and trachea in the embryo of Xenopus laevis. Am. F. Anat. 122, 19-55.

Suprenant, K. A. \& Dentler, W. L. (1986). Differential stability of microtubule-capping structures to $\mathrm{Ca}^{++}$and $\mathrm{Mg}^{++}$. F. Cell Biol. 103, 133a.

TAylor, A. C. \& Kollros, J. J. (1946). Stages in the normal development of Rana pipiens larvae. Anat. Rec. 94, 7-23. 
Venable, J. H. \& Coggeshall, R. (1965). A simplified lead citrate stain for use in electron microscopy. $\mathcal{Y}$. Cell Biol. 25, 407-408.

WITMAN, G. B. (1975). The site of in vito assembly of flagellar microtubules. Ann. N.Y. Acad. Sci. 253, 178-191.
Youson, J. H. (1982). Replication of basal bodies and ciliogenesis in a ciliated epithelium of the lamprey. Cell Tiss. Res. 223, 255-266.

(Received 26 September 1986 - Accepted 14 October 1986) 